\title{
Agentes Crioprotectores Alternativos para el Congelamiento Lento de Espermatozoides Epididimarios de Alpaca (Vicugna pacos)
}

\author{
Alternative Cryoprotectans Suitable for an Slow Freezing Method of \\ Epididymal Spermatozoa of Alpaca (Vicugna pacos)
}

Nadia Canorio Pariona ${ }^{1,2,3}$, Fernando Paredes Arnedo ${ }^{1}$, Martha Valdivia Cuya ${ }^{1}$

\section{Resumen}

\begin{abstract}
El objetivo del estudio fue utilizar agentes crioprotectores alternativos para el congelamiento lento de espermatozoides epididimarios de alpaca (Vicugna pacos). Epidídimos obtenidos en el camal de la ciudad de Huancavelica fueron transportados a $4{ }^{\circ} \mathrm{C}$ en solución fisiológica al laboratorio en Lima, Perú. Los espermatozoides fueron extraídos de la cola del epidídimo e incubados en medio HAMF10 a $37^{\circ} \mathrm{C}$. Se usó el medio Tes-Tris-Yolk como medio de criopreservación y suplementado con los agentes crioprotectores dimetilsulfóxido $\left(\mathrm{Me}_{2} \mathrm{SO}_{4}\right)$ en concentraciones de $0.5,0.25$ y $0.125 \mathrm{M}$ y dimetilacetamida (DMA) en concentraciones de $0.75,0.385$ y $0.18 \mathrm{M}$, y comparados con el grupo control criopreservado con glicerol 0.6M. Se evaluó la motilidad, viabilidad e integridad de membrana antes y después del congelamiento. Se obtuvo una mejor viabilidad e integridad de membrana posdescongelamiento con DMA $0.375 \mathrm{M}(\mathrm{p}<0.05)$. La motilidad fue mejor en los grupos de $\mathrm{Me}_{2} \mathrm{SO}_{4} 0.25$ y $05 \mathrm{M}$ y de DMA 0.375 y $0.75 \mathrm{M}$ $(\mathrm{p}<0.05)$.
\end{abstract}

Palabras clave: alpaca, espermatozoide, criopreservación, dimetilacetamida, dimetilsulfóxido

\section{Abstract}

The objective of this study was to use alternative cryoprotectans suitable for freezing epididymal spermatozoa of alpaca (Vicugna pacos) in a slow freezing method. Epididymides obtained in the slaughterhouse of Huancavelica city were transported at

${ }^{1}$ Laboratorio de Fisiología de la Reproducción Animal (LFRA), Facultad de Ciencias Biológicas, Universidad Nacional Mayor de San Marcos, Lima, Perú

${ }^{2}$ E-mail: ncanorio@gmail.com

${ }^{3}$ Fuente de financiamiento: ICGEB Grant CRP/PER03-01 Molecular analysis of alpacas gametes interactions

Recibido: 7 de enero de 2015

Aceptado para publicación: 20 de abril de 2015 
$4{ }^{\circ} \mathrm{C}$ in saline solution to the laboratory in Lima, Peru. The sperm cells were extracted from the epididymis and incubated in HAMF10 medium. The Tes-Tris-Yolk-Citrate medium was used and supplemented with the cryoprotectants dimethyl sulfoxide $\left(\mathrm{Me}_{2} \mathrm{SO}_{4}\right)$ at 0.5 , 0.25 and $0.125 \mathrm{M}$ concentrations, and dimethylacetamide (DMA) at $0.75,0.385$ and $0.18 \mathrm{M}$ concentrations, and compared with a control group cryopreserved with glycerol $0.6 \mathrm{M}$. Motility, viability and integrity of membrane before and after freezing were assessed. The results showed a better viability and membrane integrity post-freezing when using DMA $0.375 \mathrm{M}(\mathrm{p}<0.05)$. Motility was better with $\mathrm{Me}_{2} \mathrm{SO}_{4} 0.25$ and $05 \mathrm{M}$ and DMA 0.375 and $0.75 \mathrm{M}(\mathrm{p}<0.05)$.

Key words: alpaca, sperm, cryopreservation, dimethylacetamide, dimethylsulfoxide

\section{INTRODUCCIÓN}

La criopreservación del semen de alpaca es una técnica muy limitada debido a su escaso desarrollo (Bravo et al., 2000). Además, el semen es generalmente pobre en calidad y altamente viscoso, haciendo difícil su manejo, dilución y evaluación. Trabajos sobre criopreservación de semen de alpaca reportan entre 6 y $20 \%$ de motilidad posdescongelamiento (Vaughan et al., 2003; Santiani et al., 2005) y raramente superan el $40 \%$, aunque Bravo et al. (1996) reportaron $45 \%$, pero sin dar detalles que permitan la réplica de su metodología.

En años recientes se ha trabajado en la criopreservación de espermatozoides epididimarios de alpaca con mejores resultados. Morton et al. (2010) reportaron diferencias en las propiedades de la membrana plasmática entre los medios usados, especialmente debido a la fuente de la muestra (eyaculado o epidídimo).

El uso de agentes crioprotectores permeables es esencial para la viabilidad posdescongelamiento de los espermatozoides, ya que se minimiza la formación de hielo intracelular; sin embargo, la introducción y remoción de estos agentes crioprotectores pueden dañar la célula. La mayoría de trabajos sobre criopreservación de espermatozoides de alpaca ha empleado glicerol (6-7\%) como agente crioprotector, donde los resultados demuestran una recuperación pobre tras la descongelación, acompañada con la pérdida parcial de la motilidad inicial (Vaughan $e t$ al., 2003; Santiani et al., 2005; Valdivia et al., 2005). Recientemente, una metodología eficiente de criopreservación de espermatozoides epididimarios de alpaca ha sido desarrollada utilizando glicerol a una concentración de 3\% (Morton et al., 2007). A pesar de sus beneficios, el glicerol es potencialmente citotóxico a ciertas concentraciones (Almilid y Jhonson, 1988; Holt, 2000; Watson, 2000) y tiene un efecto contraceptivo en algunas especies (Sexton, 1975; Hay et al., 1997).

Son pocos los estudios que se han enfocado en crioprotectores alternativos para la criopreservación de espermatozoides de alpaca, estimulando la búsqueda de agentes como dimetilacetamida (DMA) o dimetilsulfóxido $\left(\mathrm{Me}_{2} \mathrm{SO}_{4}\right)$ (Lovelock et al., 1959; Ball et al., 2001), en consideración a su naturaleza altamente hidrofílica y su bajo tamaño molecular. Así mismo, DMA ha sido usada en la criopreservación de espermatozoides de aves (Blanco et al., 2000) y mamíferos como el koala y el canguro (McClean et al., 2008), y es el agente crioprotector más usado en la criopreservación de espermatozoides de peces (Tiersch et al., 2004; Vuthiphandchai et al., 2009; Yang et al., 2010). 
El estudio tuvo como objetivo evaluar la efectividad de DMA y $\mathrm{Me}_{2} \mathrm{SO}_{4}$ como agentes crioprotectores en el proceso de congelamiento lento de espermatozoides epididimarios de alpaca.

\section{Materiales y Métodos}

\section{Animales}

Se utilizaron 30 muestras provenientes de 30 alpacas de 4 a 7 años de edad. Los animales fueron sacrificados en el camal municipal de la ciudad de Huancavelica. Los testículos fueron colectados con el escroto completo bajo condiciones de higiene y transportados a $4-8{ }^{\circ} \mathrm{C}$ al Laboratorio de Fisiología de la Reproducción Animal (LFRA) de la Facultad de Ciencias Biológicas, Universidad Nacional Mayor de San Marcos, Lima, donde llegaron dentro de las 14 horas del sacrificio.

\section{Colección de la Muestra}

En el laboratorio se procedió a aislar la región de la cola del epidídimo, el cual fue limpiado y liberado de venas residuales y restos de sangre. Fue cortado en pedazos pequeños para permitir la salida de los espermatozoides en solución salina $(0.9 \%$ $\mathrm{NaCl}$ ) a $37{ }^{\circ} \mathrm{C}$. La suspensión de espermatozoides fue transferido a tubos plásticos de $10 \mathrm{ml}$.

Se evaluó la concentración espermática, motilidad, viabilidad e integridad de membrana. Las muestras de espermatozoides fueron centrifugadas y resuspendidas en medio HAM F10 (Sigma, Cat. N. ${ }^{\circ}$ N6635) por 30 min a $37^{\circ} \mathrm{C}$. Se recuperó el sobrenadante que contenía los espermatozoides móviles y morfológicamente normales, y se procedió a la evaluación de la concentración, viabilidad, motilidad e integridad de membrana.

\section{Evaluación Espermática}

- Concentración espermática. Se hizo una dilución de $10 \mu \mathrm{L}$ de la muestra fresca en $90 \mu \mathrm{L}$ de agua. Se colocó $10 \mu \mathrm{L}$ de esta dilución en cada cámara de un hemocitómetro (Improved Naebauer, Weber, Londres, Reino Unido). Se contaron los espermatozoides en las rejillas de cada lado de la cámara y el promedio de ambos lados fue usado para calcular el número de espermatozoides $\mathrm{ml}^{-1}$ en la muestra inicial.

- Motilidad. Se evaluó en forma subjetiva. Se colocó una alícuota de $10 \mu \mathrm{L}$ de la muestra resuspendida de espermatozoides en una lámina precalentada y cubierta con una laminilla precalentada, como describen Evans y Maxwell (1987), y se observó bajo microscopía de luz. Se expresó en porcentaje.

- Viabilidad. Se evaluó utilizando el colorante vital eosina. Se colocó una gota de muestra y una gota del colorante y se incubó a $37^{\circ} \mathrm{C}$ por $5 \mathrm{~min}$. Luego, se procedió a realizar el conteo de espermatozoides no coloreados (espermatozoides vivos). Los espermatozoides coloreados de naranja/rosa corresponden a espermatozoides muertos. Se expresó en porcentaje.

- Integridad de membrana plasmática. Se empleó el test hipoosmótico (Jeyendran et al., 1984), el cual consiste en incubar $100 \mu 1$ de la muestra en $900 \mu 1$ de medio hipoosmótico ( $7.35 \mathrm{~g}$ de citrato de sodio y $13.51 \mathrm{~g}$ de fructosa en 1000 $\mathrm{ml}, 150 \mathrm{mOsm} / \mathrm{kg}$ ). La incubación se hizo a $37{ }^{\circ} \mathrm{C}$ por $30 \mathrm{~min}$. Se evaluó por microscopía. Espermatozoides con la cola enrollada correspondían a espermatozoides con membrana plasmática intacta y espermatozoides con cola recta correspondían a espermatozoides con la membrana plasmática alterada. Se expresó en porcentaje. 


\section{Diseño Experimental}

Las concentraciones de crioprotectores usadas en este trabajo fueron realizadas en base a trabajos previos realizados en nuestro laboratorio (Valdivia et al., 2005).

En las primeras pruebas se evaluaron agentes crioprotectores como dimetilsulfóxido, propilenglicol, glicerol y dimetilacetamida en varias concentraciones con resultados desalentadores. En el caso de glicerol y propilenglicol $(1.5,1.0,0.5$ y $0.25 \mathrm{M})$ no hubo espermatozoides móviles posdescongelamiento y la viabilidad e integridad de membrana fueron inferiores al 30\% (resultados no mostrados). En el caso de dimetilsulfóxido $\left(\mathrm{Me}_{2} \mathrm{SO}_{4}\right)$ y dimetilacetamida (DMA) en concentraciones de 2.0, $1.5 \mathrm{y}$ $1.0 \mathrm{M}$, se encontró una motilidad posdescongelamiento de $10 \%$ y viabilidad e integridad de membrana cercanas al 50\%. Ante esto, se procedió a evaluar concentraciones menores.

Las muestras fueron divididas en tres grupos: Un grupo criopreservado con glicerol al 3\% (grupo control), otro criopreservado con dimetilsulfóxido $(0.5 \mathrm{M}, 0.25 \mathrm{M}$ y $0.125 \mathrm{M})$ y otro criopreservado con dimetilacetamida $(0.75 \mathrm{M}, 0.375 \mathrm{M}$ y $0.18 \mathrm{M})$. Al final, se obtuvo cuatro pajuelas de $0.25 \mathrm{ml}$ para cada concentración de cada crioprotector, incluido el grupo control.

\section{Proceso de Criopreservación}

Se usó el medio Tes-Tris-Yolk para el proceso de criopreservación, el cual incluía una solución de citrato de sodio al 5\% (Sigma, código D1546), solución de Tes al 2\% (Sigma, código T1375), Trizma Base al 8\% (Sigma, código T6066) y yema de huevo al 20\%. La solución final fue suplementada con estreptomicina y penicilina (1:1000). El medio preparado fue suplementado con los agentes crioprotectores dimetilacetamida y dimetilsulfóxido en sus diversas concentraciones y glicerol en la concentración de $0.6 \mathrm{M}$.

La muestra de espermatozoides fue ajustada a $20 \times 10^{6}$ espermatozoides $/ \mathrm{ml}$, la cual fue mezclada en una proporción de 1:1 con el medio Tes-Tris-Yolk suplementado con el agente crioprotector logrando una concentración espermática final para la criopreservación de $10 \times 10^{6}$ espermatozoides/ml. Esta dilución fue mantenida a $4{ }^{\circ} \mathrm{C}$ por una hora, lo cual correspondió al tiempo de estabilización, periodo donde hay un descenso en la temperatura de $37{ }^{\circ} \mathrm{C}$ a $4{ }^{\circ} \mathrm{C}$ a una velocidad de $0.55^{\circ} \mathrm{C} / \mathrm{min}$. Luego, las muestras fueron colocadas en pajuelas de criopreservación de $0.25 \mathrm{ml}$, selladas y colocadas en el sistema de congelamiento (CL3300, Cryologic, Australia) termocontrolado por el software Cryogenesis $4.1 \mathrm{del}$ equipo, a velocidades de $3-5{ }^{\circ} \mathrm{C} / \mathrm{min}$.

El protocolo de congelamiento fue el siguiente: La temperatura inicial de $4{ }^{\circ} \mathrm{C}$ fue mantenida por $5 \mathrm{~min}$. Luego se hizo un descenso de +4 a $-2{ }^{\circ} \mathrm{C}$ a $3{ }^{\circ} \mathrm{C} /$ min y mantenidos a $-2{ }^{\circ} \mathrm{C}$ por $1 \mathrm{~min}$. Allí se procedió a hacer la siembra (seeding), que consiste en tocar la base de las pajuelas con la punta de una pinza a $-196{ }^{\circ} \mathrm{C}$ hasta que se viera un ligero cambio de color en las paredes de las pajuelas, lo cual indica la formación de microcristales. Se continuó con el descenso de la temperatura hasta $-30{ }^{\circ} \mathrm{C}$ (velocidad de congelamiento de $5{ }^{\circ} \mathrm{C}$ por min) y de -30 a $-80{ }^{\circ} \mathrm{C}$ (velocidad de congelamiento de $\left.8{ }^{\circ} \mathrm{C} / \mathrm{min}\right)$. Finalmente, las pajuelas se colocaron en nitrógeno líquido $\left(-196{ }^{\circ} \mathrm{C}\right)$.

A las 72 h de la congelación se procedió a la descongelación de las pajuelas en baño maría a $50{ }^{\circ} \mathrm{C}$ por $7 \mathrm{~s}$. La muestra descongelada fue centrifugada y el pellet obtenido fue resuspendido en $0.5 \mathrm{~mL}$ de medio HAM F1 y mantenido a $37{ }^{\circ} \mathrm{C}$ por $10 \mathrm{~min}$. Luego se procedió a evaluar la motilidad, viabilidad e integridad de membrana en los espermatozoides. 
Cuadro 1. Resultados obtenidos después del periodo de estabilización a $4{ }^{\circ} \mathrm{C}$ por $1 \mathrm{~h}$ en espermatozoides epididimarios usando el medio Tes-Tris-Yolk suplementado con los agentes crioprotectores Dimetilacetamida (DMA) y Dimetilsulfóxido $\left(\mathrm{Me}_{2} \mathrm{SO}_{4}\right)$ a diferentes concentraciones ${ }^{1}$

\begin{tabular}{lccc}
\hline Agente crioprotector & $\begin{array}{c}\text { Motilidad } \\
(\%)\end{array}$ & $\begin{array}{c}\text { Viabilidad } \\
(\%)\end{array}$ & $\begin{array}{c}\text { Integridad de la } \\
\text { membrana plasmática } \\
(\%)\end{array}$ \\
\hline DMA 0.18M & $56.4 \pm 4.3^{\mathrm{a}}$ & $69.3 \pm 3.7^{\mathrm{a}}$ & $64.0 \pm 3.5^{\mathrm{a}}$ \\
DMA 0.375M & $55.1 \pm 3.2^{\mathrm{a}}$ & $75.2 \pm 3.0^{\mathrm{b}}$ & $72.8 \pm 3.2^{\mathrm{b}}$ \\
$\mathrm{DMA} 0.75 \mathrm{M}$ & $52.7 \pm 5.7^{\mathrm{a}}$ & $76.0 \pm 4.8^{\mathrm{b}}$ & $67.3 \pm 3.0^{\mathrm{a}}$ \\
$\mathrm{Me}_{2} \mathrm{SO}_{4} 0.125 \mathrm{M}$ & $52.5 \pm 5.9^{\mathrm{a}}$ & $68.5 \pm 4.5^{\mathrm{a}}$ & $64.2 \pm 2.8^{\mathrm{a}}$ \\
$\mathrm{Me}_{2} \mathrm{SO}_{4} 0.25 \mathrm{M}$ & $42.8 \pm 4.5^{\mathrm{b}}$ & $63.7 \pm 3.6^{\mathrm{c}}$ & $61.8 \pm 2.5^{\mathrm{a}}$ \\
$\mathrm{Me}_{2} \mathrm{SO}_{4} 0.5 \mathrm{M}$ & $34.6 \pm 4.5^{\mathrm{b}}$ & $60.7 \pm 3.4^{\mathrm{c}}$ & $63.7 \pm 3.1^{\mathrm{a}}$ \\
$\mathrm{Glicerol}^{0.6 \mathrm{M}}$ & $35.3 \pm 3.1^{\mathrm{b}}$ & $62.7 \pm 3.7^{\mathrm{c}}$ & $61.8 \pm 3.6^{\mathrm{a}}$ \\
\hline
\end{tabular}

${ }^{1}$ Los datos son presentados como promedios \pm desviaciones estándar.

$a, b, c$ Valores con superíndices diferentes dentro de columnas indican diferencias significativas $(p<0.05)$

\section{Análisis Estadístico}

Los valores obtenidos en la evaluación espermática fueron analizados mediante el análisis de varianza de un factor, luego de realizar la transformación de los datos con el arc-sen. Se rechazaron comparaciones mediante el análisis estadístico no paramétrico (Prueba de Tukey) y las diferencias fueron consideradas significativas cuando $\mathrm{p}<0.05$. Los resultados fueron expresados como media \pm desviación estándar (d.e.). Los análisis fueron realizados usando el paquete estadístico SPSS (v. 17.00 para Windows).

\section{Resultados}

El presente estudio evaluó el efecto de dos agentes crioprotectores $\left(\mathrm{Me}_{2} \mathrm{SO}_{4} \mathrm{y}\right.$ DMA) durante el proceso de criopreservación de espermatozoides epididimarios de alpaca. Los resultados fueron comparados con aque- llos obtenidos con la muestra control (criopreservados con glicerol $0.6 \mathrm{M}$ ). La muestra inicial presentó una concentración espermática promedio de $56 \times 10^{6}$ espermatozoides $/ \mathrm{ml}$, motilidad de $77.3 \pm 5 \%$, viabilidad de $72.5 \pm 4.2 \%$ e integridad de membrana plasmática de $63.2 \pm 5.2 \%$.

Los resultados de motilidad, viabilidad e integridad de la membrana plasmática luego del proceso de estabilización a $4{ }^{\circ} \mathrm{C}$ por $1 \mathrm{~h}$ indican que se obtuvieron mejores valores con el uso de DMA en sus tres concentraciones ( $\mathrm{p}<0.05$; Cuadro 1).

En el Cuadro 2 se presentan los resultados luego del proceso de congelación y descongelación. La viabilidad e integridad de membrana fueron mejores cuando se usó DMA 0.375M. En el caso de la motilidad, los resultados fueron bastante similares, con excepción de $\mathrm{Me}_{2} \mathrm{SO}_{4} 0.125 \mathrm{M}$, DMA $0.18 \mathrm{M}$ y glicerol $0.6 \mathrm{M}$ donde los valores fueron los más bajos $(\mathrm{p}<0.05)$. 
Cuadro 2. Resultados obtenidos luego del proceso de descongelamiento en espermatozoides epididimarios usando el medio Tes-Tris-Yolk suplementado con los agentes crioprotectores Dimetilacetamida (DMA) y Dimetilsulfóxido $\left(\mathrm{Me}_{2} \mathrm{SO}_{4}\right)$ a diferentes concentraciones ${ }^{1}$

\begin{tabular}{lccc}
\hline Agente crioprotector & $\begin{array}{c}\text { Motilidad } \\
(\%)\end{array}$ & $\begin{array}{c}\text { Viabilidad } \\
(\%)\end{array}$ & $\begin{array}{c}\text { Integridad de la membrana } \\
\text { plasmática } \\
(\%)\end{array}$ \\
\hline DMA 0.18M & $27.0 \pm 1.4^{\mathrm{a}}$ & $40.5 \pm 2.6^{\mathrm{a}}$ & $45.4 \pm 12.0^{\mathrm{a}}$ \\
DMA 0.375M & $34.4 \pm 1.4^{\mathrm{b}}$ & $60.9 \pm 4.0^{\mathrm{b}}$ & $64.4 \pm 1.7^{\mathrm{b}}$ \\
DMA 0.75M & $28.6 \pm 4.7^{\mathrm{b}}$ & $45.7 \pm 1.8^{\mathrm{c}}$ & $52.5 \pm 8.2^{\mathrm{c}}$ \\
$\mathrm{Me}_{2} \mathrm{SO}_{4} 0.125 \mathrm{M}$ & $22.2 \pm 6.1^{\mathrm{a}}$ & $46.9 \pm 6.8^{\mathrm{d}}$ & $46.2 \pm 11.8^{\mathrm{a}}$ \\
$\mathrm{Me}_{2} \mathrm{SO}_{4} 0.25 \mathrm{M}$ & $33.3 \pm 4.9^{\mathrm{b}}$ & $48.7 \pm 2.2^{\mathrm{d}}$ & $52.2 \pm 1.0^{\mathrm{d}}$ \\
$\mathrm{Me}_{2} \mathrm{SO}_{4} 0.5 \mathrm{M}$ & $27.9 \pm 5.3^{\mathrm{b}}$ & $50.3 \pm 5.0^{\mathrm{d}}$ & $51.6 \pm 4.3^{\mathrm{c}}$ \\
$\mathrm{Glicerol} \mathrm{0.6M}$ & $17.6 \pm 2.7^{\mathrm{a}}$ & $35.5 \pm 3.8^{\mathrm{e}}$ & $40.5 \pm 2.7^{\mathrm{a}}$ \\
\hline
\end{tabular}

${ }^{1}$ Los datosson presentados como promedios \pm desviaciones estándar.

$a, b, c$ Valores con superíndices diferentes dentro de columnas indican diferencias significativas $(p<0.05)$

\section{Discusión}

La criopreservación de semen de alpaca ha tenido un progreso pobre y desalentador comparado con otras especies domesticas (Vaughan et al., 2003; Santiani et al., 2005). El uso reciente de espermatozoides del epidídimo para desarrollar técnicas de congelamiento de semen en alpacas han cambiado la percepción sobre el espermatozoide de alpaca y brinda luces en la fisiología de esta célula. En el presente trabajo se evaluaron dos nuevos agentes crioprotectores, dimetilacetamida y dimetilsulfóxido, en el congelamiento lento de espermatozoides epididimarios de alpaca.

Se trabajó con el medio Tes-Tris-Yolk para el proceso de criopreservación, el cual ha sido usado en otras especies, como en toro (Vishwanath y Shannon, 2000), carnero (Evans y Maxwell, 1987), cabra (Leboeuf et al., 2000), alpaca (Vaughan et al., 2003), 1la- ma (Ratto et al., 1999) y camello (NiasariNaslaji et al., 2006). Por otro lado, Wani et al. (2005) reportaron mejores resultados de viabilidad usando Tes-Tris-Yolk y TrisLactosa-Yolk en comparación con los medios citrato yolk, sucrosa yolk and Trisfructosa yolk en semen de camellos.

La velocidad de enfriamiento empleada fue entre $4-7^{\circ} \mathrm{C} / \mathrm{min}$, la cual es ideal para estos agentes crioprotectores. Asimismo, los cuatro puntos de temperatura de referencia durante el enfriamiento $\left(+4,-2,-30\right.$ y $\left.-80{ }^{\circ} \mathrm{C}\right)$ son puntos estándares en las curvas de enfriamiento lento que se emplean en otras especies (Willadsen et al., 1976; Curry et al., 1994). Por otro lado, el ambiente intra y extracelular es muy diferente cuando el espermatozoide llega a $-196{ }^{\circ} \mathrm{C}$, pudiendo ocurrir daño si la velocidad de descongelación no es compatible con la velocidad de congelación, de allí que se haya usado un descongelamiento rápido $\left(50^{\circ} \mathrm{C}\right.$ por $\left.7 \mathrm{~s}\right)$. 
Glicerol al 3\% es el agente crioprotector más usado para criopreservar espermatozoides de alpaca (Morton et al., 2007), pero puede producir efectos tóxicos y contraceptivos en espermatozoides criopreservados de otras especies (Holt et al., 2000); asimismo, su comportamiento durante el congelamiento depende de la velocidad de enfriamiento. En los preliminares del presente estudio se utilizaron varias concentraciones $(1.5,1.0,0.5 \mathrm{y}$ $0.25 \mathrm{M}$ ) con resultados posdescongelamiento inadecuados (resultados no mostrados), lo cual posiblemente se debía al comportamiento molecular del glicerol, ya que es un buen agente crioprotector en procesos de congelamiento rápido debido a la alta viscosidad que muestra, donde la velocidad de enfriamiento óptima sería de $10-50{ }^{\circ} \mathrm{C} / \mathrm{min}$.

Crioprotectores como DMA y $\mathrm{Me}_{2} \mathrm{SO}_{4}$ (Lovelock et al., 1959; Ball et al., 2001; Alvarenga et al., 2005) son buenos candidatos debido a su naturaleza altamente hidrofílica, bajo peso molecular y nivel de toxicidad inferior al del glicerol. DMA reduce la formación de cristales de hielo intracelular e incrementa la permeabilidad de la membrana decreciendo el daño osmótico (Ball et al., 2001), en tanto que la penetración del $\mathrm{Me}_{2} \mathrm{SO}$ es también rápida debido a su bajo peso molecular (Lovelock et al., 1959) y ha sido usado con éxito en varias especies (Kundu et al., 2000).

Las concentraciones de DMA empleadas en el presente estudio fueron evaluadas en otras especies, como conejo (Dalimata y Graham, 1997, Okuda et al., 2007), jabalí (Kim et al., 2011), tortuga y peces (Tiersch et al., 2004; Vuthiphandchai et al., 2009; Yang et al., 2010), canguro (McClean et al., 2008), caballo (Medeiros et al., 2002, Squires et al., 2004) y koala (Zee et al., 2008; Johnston et $a l ., 2012$ ), donde emplearon concentraciones de 0.25 a $1.25 \mathrm{M}$, reportando valores posdescongelamiento de $45 \%$ de motilidad, $58 \%$ de viabilidad y $60 \%$ de integridad de membrana plasmática.
El $\mathrm{Me}_{2} \mathrm{SO}$ ha sido usado en diversas especies como cabra (Kundu et al., 2000), lobo colorado (Johnson et al., 2014), peces (Anchordoguya et al., 1988; Thirumala et al., 2005, 2006; Vuthiphandchai et al., 2009; Sanches et al., 2013), cerdo (Hood et al., 1970), caballo (Devireddy et al., 2002) y humano (Serafini et al., 1986), en concentraciones de 0.25 a $1.5 \mathrm{M}$, encontrando valores posdescongelamiento de $35 \%$ de motilidad, $59 \%$ de viabilidad y $50 \%$ de integridad de membrana.

Los resultados posdescongelamiento muestran un descenso en los parámetros de motilidad, viabilidad e integridad de membrana, siendo la motilidad la más afectada, como lo reportan otros autores (Watson, 1995). Los valores más altos de motilidad obtenidos en el presente estudio concuerda con las concentraciones empleadas para $\mathrm{Me}_{2} \mathrm{SO}_{4}$ y DMA en otros estudios (Kundu et al., 2000; Devireddy et al., 2002; Medeiros et al., 2002; Squires et al., 2004; Tiersch et al., 2004; Thirumala et al., 2006; Okuda et al., 2007; McClean et al., 2008; Vuthiphandchai et al., 2009; Yang et al., 2010; Kim et al., 2011; Sanches et al., 2013; Johnson et al., 2014).

\section{Conclusiones}

- La motilidad, viabilidad e integridad de membrana del espermatozoide epididimario de la alpaca se ven afectadas durante el proceso de congelamiento, siendo la motilidad el parámetro más afectado ( $\mathrm{p}<0.05)$.

- Luego del proceso de congelación y descongelación se obtuvieron mejores valores de viabilidad e integridad de la membrana plasmática cuando se usó DMA $0.375 \mathrm{M}(\mathrm{p}<0.05)$.

- Los agentes crioprotectores dimetilsulfóxido $(0.75 \mathrm{M}$ y $0-375 \mathrm{M})$ y dimetilacetamida $(0.5 \mathrm{M}$ y $0.25 \mathrm{M})$ son buenos candidatos para ser usados en los procedimientos de congelamiento lento de espermatozoides epididimarios de alpa- 
ca, ofreciendo mejores resultados posdescongelamiento en comparación con el glicerol $(0.6 \mathrm{M})$.

\section{Literatura Citada}

1. Almilid T, Johnson LA. 1988. Effects of glycerol concentration, equilibration time and temperature of glycerol addition on post-thaw viability of boar spermatozoa frozen in straws. J Anim Sci 66: 2899-2905.

2. Alvarenga M, Papa FO, LandimAlvarenga FC, Medeiros ASL. 2005. Amides as cryoprotectants for freezing stallion semen: a review. Anim Reprod Sci 89: 105-113.

3. Anchordoguy T, Crowe J, Griffin F, Clark W. 1988. Cryopreservation of sperm from the marine shrimp Sicyonia ingentis. Cryobiology 25: 238-243.

4. Ball BA, Medina V, Gravance CG, Baumbe J. 2001. Effect of antioxidants on preservation of motility, viability and acrosomal integrity of equine spermatozoa during storage at $5^{\circ} \mathrm{C}$. Theriogenology 56: 577-589.

5. Blanco J, Gee G, Wildt D, Donoghue A. 2000. Species variation in osmotic, cryoprotectant, and cooling rate tolerance in poultry, eagle, and peregrine falcon spermatozoa. Biol Reprod 63: 1164-1171. doi: 10.1095/ biolreprod63.4.1164

6. Bravo PW, Ccallo M, Garnica J. 2000. The effect of enzymes on semen viscosity in llamas and alpacas. Small Rumin Res 38: 91-95.

7. Bravo PW, Ordoñez C, Alarcón V. 1996. Semen processing and freezing of alpacas and llamas. In: $13^{\text {th }}$ ICAR. Sydney, Australia.

8. Curry MR, Millar JD, Watson PF. 1994. Calculated optimal cooling rates for ram and human sperm cryopreservation fail to conform with empirical observations. Biol Reprod 51: 1014-1021.
9. Dalimata AM, Graham JK. 1997. Cryopreservation of rabbit spermatozoa using acetamide in combination with trehalose and methylcellulose. Theriogenology 49: 831-841.

10. Devireddy RV, Swanlund DJ, Olin T, Vincente W, Troedsson MH, Bischof JC, Roberts KP. 2002. Cryopreservation of equine sperm: optimal cooling rates in the presence and absence of cryoprotective agents determined using differential scanning calorimetry. Biol Reprod 66: 222-231. doi: 10.1095/ biolreprod66.1.222

11. Evans G, Maxwell WMC. 1987. Salamon's artificial insemination of sheep and goats. Sydney, Australia: Butterworths. $194 \mathrm{p}$.

12. Hay MA, King WA, Gartley CJ, Leibo SP, Goodrowe KL. 1997. Effects of cooling, freezing and glycerol on penetration of oocytes by spermatozoa in dogs. J Reprod Fertil 51: 99-108.

13. Holt WV. 2000. Basic aspects of frozen storage of semen. Anim Reprod Sci 62: 3-22.

14. Hood RD, Foley CW, Martin TG 1970. Effects of cold shock, dilution, glycerol and dimethyl sulfoxide on cation concentrations in porcine spermatozoa. J Anim Sci 30: 91-94.

15. Jeyendran RS, Van der Ven HH, Perez-Pelaez M, Crabo BG, Zaneveld LJ. 1984. Development of an assay to assess functional integrity of the human sperm membrane and its relationship to other sperm characteristics. J Reprod Fertil 70: 219-228.

16. Johnson AE, Freeman EW, Wildt DE, Songsasen N. 2014. Spermatozoa from the maned wolf (Chrysocyon brachyurus) display typical canid hypersensitivity to osmotic and freezinginduced injury, but respond favorably to dimethyl sulfoxide. Cryobiology 68: 361370. doi: 10.1016/j.cryobiol.2014.04.004

17. Johnston SD, Zee YP, LópezFernández C, Gosálvez J. 2012. The effect of chilled storage and cryopreser- 
vation on the sperm DNA fragmentation dynamics of a captive population of koalas. J Androl 33: 1007-1015. doi: 10.2164/jandrol.111.015248

18. Kim S, Lee YJ, Ji DB, Kim YJ. 2011. Evaluation of different cryoprotectants (CPAs) in boar semen cryopreservation. J Vet Med Sci 73: 961-963. doi: 10.1292/ jvms.10-0345

19. Kundu CN, Chakraborty J, Dutta P, Bhattacharyya D, Ghosh A, Majumder GC. 2000. Development of a simple sperm cryopreservation model using a chemically defined medium and goat cauda epididymal spermatozoa. Cryobiology 40: 117-125. doi: 10.1006/ cryo. 2000.2230

20. Leboeuf B, Restall B, Salamon S. 2000. Production and storage of goat semen for artificial insemination. Anim Reprod Sci 62: 113-141.

21. Lovelock JE, Bishop MW. 1959. Prevention of freezing damage to living cells by dimethyl sulphoxide. Nature 183:1394-1395. doi: 10.1038/1831394a0

22. McClean R, Zee YP, Holt WW, Johnston SD. 2008. Cryopreservation of kangaroo spermatozoa using alternative approaches that reduce cytotoxic exposure to glycerol. Cryobiology 57: 304-347. doi: 10.1016/ j.cryobiol.2008.08.007

23. Medeiros ASL, Gomes GM, Carmo MT, Papa FO, Alvarenga MA. 2002. Cryopreservation of stallion sperm using different amides. Theriogenology 58: 273-276.

24. Morton K, Bathgate R, Evans $G$, Maxwell W. 2007. Cryopreservation of epididymal alpaca (Vicugna pacos) sperm: a comparison of citrate-, Tris- and lactose-based diluents and pellets and straws. Reprod Fertil Dev 19: 792-796.

25. Morton K, Evans G, Maxwell W. 2010. Effect of glycerol concentration, Equex STM supplementation and liquid storage prior to freezing on the motility and acrosome integrity of frozen-thawed epididymal alpaca (Vicugna pacos) sperm. Theriogenology 74: 311-316. doi: 10.1016/j.theriogenology.2010.02.015

26. Niasari-Naslaji A, Mosaferi $S$, Bahmani N, Gharahdaghi A, Abarghani A, Ghanbari A, Gerami A. 2006. Effectiveness of a tris-based extender (SHOTOR diluent) for the preservation of Bactrian camel (Camelus bactrianus) semen. Cryobiology 53: 12-21.

27. Okuda Y, Seita Y, Hisamatsu S, Sonoki M, Shino M, Masaoka T, Inomata T, et al. 2007. Fertility of spermatozoa cryopreserved with $2 \%$ acetamide or glycerol through artificial insemination in the Japanese white rabbit. Exp Anim 56: 29-34. doi: 10.1538/ expanim.56.29

28. Ratto MH, Wolter M, Berland M. 1999. Refrigeration of epididymal sperm from lama with three different extenders. Proc II Congreso Mundial sobre Camélidos. Cusco, Perú.

29. Sanches EG, Oliveira IR, Serralheiro PC, Cerqueira VR. 2013. Cryopreservation of mutton snapper (Lutjanus analis) sperm. An Acad Bras Cienc 85: 1083-1091. doi: 10.1590/S000137652013005000047

30. Santiani A, Huanca W. Sapana $R$, Huanca T, Sepúlveda N, Sánchez R. 2005. Effects on the quality of frozenthawed alpaca (Lama pacos) semen using two different cryoprotectants and extenders. Asian J Androl 7: 303-309.

31. Serafini PC, Hauser D, Moyer D, Marrs RP. 1986. Cryopreservation of human spermatozoa: correlations of ultrastructural sperm head configuration with sperm motility and ability to penetrate zona-free hamster ova. Fertil Steril 46: 691-695. doi: 10.1016/00207292(87)90355-9

32. Sexton TJ. 1975. Relationship of the method of addition and temperature of cryoprotective agents to the fertilizing capacity of cooled chicken spermatozoa. 
Poultry Sci 54: 845-848. doi: 10.3382/ ps.0540845

33. Squires EL, Keith SL, Graham JK. 2004. Evaluation of alternative cryoprotectants for preserving stallion spermatozoa. Theriogenology 62: 10561065.

34. Thirumala $S$, Huang $C$, Dong $Q$, Tiersch T, Devireddy R. 2005. A theoretically estimated optimal cooling rate for the cryopreservation of sperm cells from a live-bearing fish, the green swordtail Xiphophorus helleri. Theriogenology 63: 2395-2415.

35. Thirumala S, Campbell WT, Vicknair MR, Tiersch TR, Devireddy RV. 2006. Freezing response and optimal cooling rates for cryopreserving sperm cells of striped bass, Morone saxatilis. Theriogenology 66: 964-973. doi: 10.1016/j.theriogenology.2006.02.035

36. Tiersch TR, Figiel CR, Wayman WR, Williamson JH, Gorman OT, Carmichael GJ. 2004. Cryopreservation of sperm from the endangered colorado pikeminnow. N Am J Aquacult 66: 8-14. doi: 10.1577/C02-043

37. Valdivia M, Canorio N, Carrillo N, Uipan P. 2005. Effects of cryoprotectans on alpaca's spermatozoa during cooling process. Biol Reprod 73: 228.

38. Vaughan JL, Galloway D, Hopkins D. 2003. Artificial insemination in alpacas (Lama pacos). Kingston, Australia: Rural Industries Research and Development Corporation. $90 \mathrm{p}$.

39. Vishwanath R, Shannon P. 2000. Storage of bovine semen in liquid and frozen state. Anim Reprod Sci 62: 23-53.
40. Vuthiphandchai V, Chomphuthawach S, Nimrat S. 2009. Cryopreservation of red snapper (Lutjanus argentimaculatus) sperm: effect of cryoprotectants and cooling rates on sperm motility, sperm viability, and fertilization capacity. Theriogenology 72: 129-138. doi: 10.1016/j.theriogenology.2009.02.013

41. Wani $N$, Nowshari M, Wernery $U$. 2005. Storage of camel (Camelus dromedarius) epididymal spermatozoa in different extenders and at different temperatures. Biol Reprod 73: 693.

42. Watson PF. 1995. Recent developments and concepts in the cryopreservation of spermatozoa and the assessment of their post-thawing function, Reprod Fertil Dev 7: 871-891.

43. Watson PF. 2000. The causes of reduced fertility with cryopreserved semen. Anim Reprod Sci 60: 481-492.

44. Willadsen SM, Polge C, Rowson LEA, Moor RM. 1976. Deep freezing of sheep embryos. J Reprod Fertil 46: 151154.

45. Yang $H$, Norris $M$, Winn $R$, Tiersch TR. 2010. Evaluation of cryoprotectant and cooling rate for sperm cryopreservation in the euryhaline fish medaka Oryzias latipes. Cryobiology 61: 211-219. doi: 10.1016/j.cryobiol. 2010.07.006

46. Zee YP, Holt WV, Gosalvez J, Allen $C D$, Nicolson V, Pyne M, Burridge $M$, et al. 2008. Dimethylacetamide can be used as an alternative to glycerol for the successful cryopreservation of koala (Phascolarctos cinereus) spermatozoa. Reprod Fertil Dev 20: 724-733. 